\title{
Nivel de conocimiento sobre el Proceso de Atención de Enfermería (PAE) en el cuidado de la salud espiritual de los profesionales de Enfermería que laboran en las unidades críticas del Hospital Nacional Hipólito Unanue, Lima 2010
}

\author{
Level of knowledge of the process of nursing care (PAE) in caring for the spiritual health of nurses \\ working in critical units of the Hipólito Unanue National Hospital, Lima 2010.
}

María Magdalena Díaz Orihuela ${ }^{1}$ Nira Cutipa Gonzáles², Rosa Matos Salgado³

\begin{abstract}
RESUMEN
Objetivo: Determinar el nivel de conocimiento sobre el Proceso de Atención de Enfermería (PAE) en el cuidado de la salud espiritual que posee el profesional de enfermería que labora en las unidades críticas: Cirugía, Emergencia y UCI del Hospital Hipólito Unanue", Lima, 2010. Material y métodos: El estudio fue de diseño no experimental, de enfoque cuantitativo, de tipo descriptivo transversal. La muestra estuvo conformada por 40 enfermeras que laboran en las unidades críticas del Hospital Hipólito Unanue, determinada mediante un muestreo probabilístico aleatorio. Para la recolección de datos se utilizó como instrumento el cuestionario para medir el nivel de conocimiento de los enfermeros sobre el PAE del cuidado de la salud espiritual, el cual fue elaborado por las investigadoras. El instrumento fue sometido a juicio de expertos y a la prueba de confiabilidad de Alpha de Cronbach (0.73). El análisis e interpretación de los mismos se realizó utilizando el paquete estadístico SPSS v. 15. Resultados: Los resultados obtenidos, en cuanto al nivel de conocimiento sobre el PAE del cuidado de la salud espiritual fueron: un $57.5 \%$ muestra un nivel de conocimiento deficiente, un $27.5 \%$ un nivel regular, un $12.5 \%$ un nivel bueno y solo un $2.5 \%$ un nivel excelente, esto indica que la mayoría de enfermeras encuestadas tiene un nivel conocimiento deficiente sobre el PAE en el cuidado espiritual. Conclusión: La mayoría de los enfermeros desconoce el fundamento científico del PAE sobre el cuidado de la salud espiritual, siendo esto una limitante para brindar los cuidados de Enfermería de forma integral.
\end{abstract}

Palabras clave: Conocimientos, proceso de atención en enfermería, cuidado, salud espiritual.

\begin{abstract}
Objective: To determine the level of knowledge about Nursing Care Process (SAP) in the care of the spiritual health that owns the nurse working in critical units: Surgery, Emergency and ICU Hospital Hipólito Unanue “, Lima, 2010. Methods: The study was non-experimental design, quantitative approach, descriptive cross. The sample consisted of 40 nurses working in critical units Hipolito Unanue Hospital, determined by random probability sampling. For data collection instrument used questionnaire to measure the level of knowledge of nurses on the care PAE spiritual health, which was developed by the researchers. The instrument was subjected to expert judgment and test Cronbach Alpha reliability (0.73). The analysis and interpretation of data was performed using the statistical package SPSS v. 15. Results: The results obtained in the level of knowledge about the care PAE spiritual health were: $57.5 \%$ shows a poor level of knowledge, a regular level $27.5 \%, 12.5 \%$ a solid level and only $2.5 \%$ an excellent level, this indicates that the majority of surveyed nurses have poor knowledge level about PAE in spiritual care. Conclusion: Most of the nurses know the scientific basis of PAE on spiritual health care, this being a constraint to provide nursing care holistically.
\end{abstract}

Keywords: Knowledge, process of care in nursing, care, spiritual health.

1 Docente y Coordinadora de las Especialidades en Enfermería de la UPG de Ciencias de la Salud, Lima. Perú

2 Docente y Directora Académica de la EAP Enfermería, Universidad Peruana Unión, Lima. Perú

3Enfermera especialista en Cuidados Intensivos, Servicio de Emergencias, Hospital Hipólito Unánue, El Agustino, Perú 
Nivel de conocimiento sobre el Proceso de Atención de Enfermería (PAE) en el cuidado de la salud espiritual de los profesionales de Enfermería que laboran en las unidades críticas del Hospital Nacional Hipólito Unanue, Lima 2010

\section{INTRODUCCIÓN}

La profesión de enfermería, a través del tiempo y las épocas, se ha caracterizado por ser una profesión de servicio y cuidado al paciente. En consecuencia, el profesional de la salud debe mostrar un alto sentido de compromiso, lealtad, valores y humanismo en la aplicación del cuidado a todo ser humano que así lo requiera. Como parte del cuidado que todo profesional de enfermería debe ofrecer, es el cuidado espiritual bajo el enfoque holístico o integral (Collado, 2010). Asimismo, según (Pinedo, Rebolledo \& Siles, 2009), la enfermera sabe que los estados emocionales del paciente y más concretamente su estado mental ejercen un efecto sobre su cuerpo, e intenta utilizar este recurso para dar una solución a la hora de planificar el plan de cuidados, siendo el área espiritual un componente importante en la ejecución de los cuidados.

En este contexto profesional, se han realizado muchas investigaciones sobre el aspecto espiritual de la atención de enfermería. Tal es el caso de (Mc Sherry, (1998); citado por (Collado, 2010), quien realizó un estudio en el cual analizó cómo 548 enfermeras percibían la espiritualidad y proveían cuidado espiritual en la práctica clínica. Los resultados indicaron que el $71.4 \%$ identificó en el paciente la necesidad espiritual, aunque solo $39.9 \%$ se sentían capacitadas para satisfacer estas necesidades. La realidad que se enfrenta en los escenarios de cuidado es que muchas veces el cuidado espiritual es delegado al sacerdote, pastor, ministros, dejándose a un lado la participación del personal de enfermería en el mismo, ya sea porque no se sienten capacitados para ofrecer el cuidado o porque no tiene el tiempo suficiente para proveer este aspecto del cuidado. Esto revela la necesidad de un conocimiento adecuado en la enfermera sobre su rol en el cuidado espiritual, especialmente en los momentos de dolor o pérdida.

Se asume, por lo descrito anteriormente, que el cuidado espiritual no es una opción, sino un elemento constitutivo del cuidado, sin embargo, en la práctica los enfermeros tienden a pasar por alto este importante aspecto, tal es así que Calabria y Macrae, citado por (Potter, 2004) mencionan: "Es demasiado frecuente en enfermería comprobar que los profesionales no ponen demasiado énfasis en la dimensión espiritual de la naturaleza humana...". Por lo tanto, el cuidado espiritual debe ser ofrecido al paciente en todo momento, con un alto sentido de responsabilidad, dedicación y respeto a todo ser humano que así lo necesite.

Además, este cuidado debe ser planificado de acuerdo con las necesidades, previamente identificadas, durante la fase de estimado y valoración (Collado, 2010). El cuidado espiritual, como las demás áreas, deben ser afrontadas de manera racional y metodológica a través de la aplicación del Proceso de Atención de Enfermería.

Por lo tanto, se hace necesario que así como la enfermera es preparada para los diferentes aspectos de la atención del cuidado de los pacientes, también sea capacitada en el conocimiento del aspecto espiritual para que pueda brindar soporte en el cuidado espiritual del paciente. (Zevallos., 2014) plantea que "la enfermera requiere complementar su habilidad profesional en solucionar problemas, como tener pensamiento crítico, intencionalidad de dar cuidado, y conocer algunas particularidades del cuidado espiritual como el trabajo de fortalecer la esperanza, comprender qué es el cuidado, tener destrezas en la comunicación frente a esta situación de vida y dar manejo integral".

La problemática expuesta anteriormente se evidencia en las unidades críticas tales como: cirugía, emergencia y UCI del Hospital Hipólito Unanue, áreas donde los profesionales de enfermería son los encargados de brindar el cuidado al paciente.

Una de las investigadoras en el cumplimiento de su rol asistencial, como enfermera de dicho servicio pudo observar que no se emplea momentos para la oración, rezar, lecturas bíblicas, fomentar la visita del líder religioso, brindar palabras de esperanza y aliento.

Por lo tanto, este trabajo de investigación plantea el siguiente objetivo: Determinar el nivel de conocimiento sobre el Proceso de Atención de Enfermería en el cuidado de la salud espiritual que posee el profesional de enfermería que labora en las unidades críticas: Cirugía, Emergencia y UCI del Hospital Hipólito Unanue.

El objetivo específico planteado fue: Determinar el nivel de conocimiento sobre el Proceso de Atención de Enfermería en el cuidado de la salud espiritual que posee el profesional de enfermería que labora en las unidades críticas: Cirugía, Emergencia y UCI del Hospital Hipólito Unanue", Lima, 2010.

La contribución más significativa de los resultados de esta investigación constituye en plantear la necesidad de sensibilización en los enfermeros de los servicios mencionados, comprendiendo el impacto de los cuidados espirituales en los pacientes. Asimismo, esta aventura intelectual confirma el constructo teórico de la tarea profesional que sigue, metodológicamente, 
el proceso de atención de enfermería. Considerando que el cuidado de la salud espiritual forma parte del cuidado integral de las personas en estado crítico.

\section{MATERIAL Y MÉTODOS}

El presente estudio de investigación se llevó a cabo en las unidades críticas del Hospital Hipólito Unanue ubicado en el distrito de El Agustino, que es uno de los 43 distritos que integra la capital del Perú, se encuentra localizado en el área central de Lima Metropolitana, limita por el norte con los distritos de San Juan de Lurigancho, al este con el distrito de Ate y el distrito de Santa Anita.

La población está compuesta por las enfermeras que laboran en las siguientes unidades críticas: Cirugía que cuenta con un total de 39 enfermeras, la Unidad de Cuidados Intensivos con un total de 17 enfermeras y la Unidad de Emergencia que cuenta con un total de 48 enfermeras.

La muestra estuvo conformada por 40 enfermeras asistenciales elegidos por muestreo probabilístico aleatorio. Se consideró los siguientes criterios de inclusión: Todas las enfermeras que trabajan en las unidades críticas: Cirugía, UCI, y Emergencia del Hospital Hipólito Unanue, las enfermeras nombradas. Contratadas por CAAS, las enfermeras que laboran más de un año en el servicio. Los criterios de exclusión considerados son: Todas las enfermeras que laboran menos de un año en el departamento y las enfermeras que trabajan en otros servicios.

El estudio fue de diseño no experimental, puesto que se recogió la información y en una base de datos y, a partir de ello, se analizaron los resultados. Hernández, Fernández y Baptista (2006) definen este diseño como aquel estudio que se realiza sin la manipulación deliberada de sus variables. Asimismo, el estudio es de enfoque cuantitativo porque mide el nivel de conocimiento sobre el PAE en el cuidado de la salud espiritual. La investigación es de tipo descriptivo, porque se describirá los resultados del nivel de conocimiento y es de corte transversal, porque se recogieron los datos en un determinado momento, en un tiempo único.

El instrumento de recolección de datos para medir el nivel de conocimiento, sobre el PAE del cuidado de la salud espiritual, se empleó un instrumento denominado "cuestionario de conocimiento" de selección múltiple elaborado por las investigadoras, la primera parte incluía los datos generales tales como la edad, el sexo, religión, grado alcanzado, años de experiencia laboral, servicio en que trabaja; la segunda parte consta de datos específicos cuyos ítemes abordaron preguntas sobre el conocimiento de las enfermeras sobre el proceso de atención de enfermería en el cuidado de la salud espiritual que consta de 34 preguntas, distribuidas de la siguiente manera: 6 preguntas de información general, 12 preguntas de conocimientos sobre conceptos generales del cuidado de la salud espiritual y 16 preguntas del Proceso de Atención de Enfermería de la salud espiritual. El instrumento fue validado por 5 jueces especializados en el área, para la prueba de fiabilidad del instrumento se aplicó una prueba piloto a una muestra con similares características, para determinar la fiabilidad y consistencia interna del instrumento se utilizó el coeficiente de Alpha de Cronbach, arrojando un coeficiente total de 0.737 valorado como una alta fiabilidad, la cual indica que hay buena relación entre los ítemes.

Se midió la consistencia interna de cada componente de la encuesta, obteniendo los siguientes resultados: conceptos generales del cuidado de la salud espiritual: 0, 682, PAE en la salud espiritual: 0,534 , se concluyó que el instrumento utilizado en la investigación presenta buen nivel de fiabilidad.

Para el análisis de los resultados, se utilizó tablas y gráficos unidimensionales y bidimensionales, así como el uso de una estadística para variable descriptiva.

Una vez obtenido los datos, estos fueron procesados usando el software SPSS versión 15, mediante el cual se procedió a realizar el análisis de los resultados.

\section{RESULTADOS}

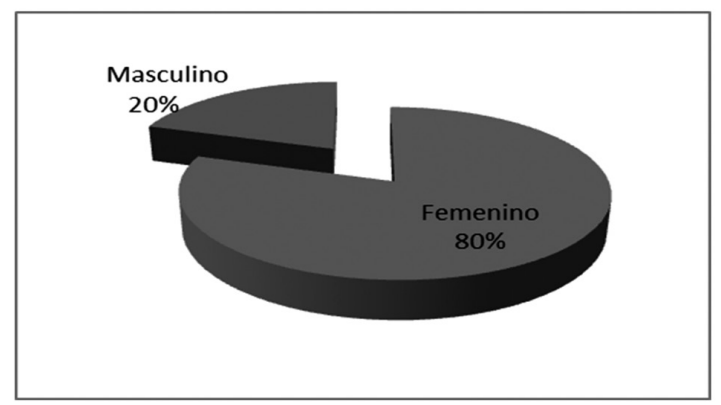

Figura 1

Género de los profesionales de enfermería que laboran en las unidades críticas del Hospital Hipólito Unanue, 2013. 
Nivel de conocimiento sobre el Proceso de Atención de Enfermería (PAE) en el cuidado de la salud espiritual de los profesionales de Enfermería que laboran en las unidades críticas del Hospital Nacional Hipólito Unanue, Lima 2010

En la figura 1 se muestra que del total de profesionales de enfermería que fueron encuestados, el $80 \%$ son de género femenino y solo el $20 \%$ son de género masculino, esto indica que la mayoría de las encuestadas son mujeres.

Tabla 1

Distribución de las enfermeras asistenciales por servicios del Hospital Hipólito Unánue, 2010.

\begin{tabular}{lll}
\hline Servicio & $\mathrm{N}^{\circ}$ & $\%$ \\
\hline Emergencia & 15 & $37,5 \%$ \\
Cirugía & 18 & $45 \%$ \\
UCl & 7 & $17,5 \%$ \\
Total & 40 & $100 \%$ \\
\hline
\end{tabular}

En la tabla 1, de $100 \%$ de enfermeras encuestadas, un $37.5 \%$ trabajan en el servicio de Emergencia, un $45 \%$ del servicio de Cirugía y un $17.5 \%$ labora en el servicio de Cuidados Intensivos, esto indica que la mayoría de encuestadas son del servicio de Cirugía.

Tabla 2

Nivel de conocimiento de los profesionales de Enfermería que laboran en las Unidades Críticas del Hospital Hipólito Unanue.

\begin{tabular}{llllll}
\hline & & \multicolumn{4}{c}{ Unidades Críticas } \\
\cline { 3 - 6 } & & Emergencia & Cirugía & UCI & Total \\
\hline & Deficiente: & 13 & 7 & 3 & 23 \\
& menor de 10 & $32,50 \%$ & $17,50 \%$ & $7,50 \%$ & $57,50 \%$ \\
Nivel de & Regular:11-13 & 1 & 6 & 4 & 11 \\
conocimiento & & $2,50 \%$ & $15,00 \%$ & $10,00 \%$ & $27,50 \%$ \\
& \multirow{2}{*}{ Buena:14-16 } & 0 & 5 & 0 & 5 \\
& & $0,00 \%$ & $12,50 \%$ & $0,00 \%$ & $12,50 \%$ \\
& Excelente:18-20 & 0 & 1 & 0 & 1 \\
& & $0,00 \%$ & $2,50 \%$ & $0,00 \%$ & $2,50 \%$ \\
\hline Total & & 14 & 19 & 7 & 40 \\
\hline & & $47,50 \%$ & $17,50 \%$ & $100,00 \%$ & \\
\hline
\end{tabular}

En la tabla 2 se observa que del $100 \%$ de profesionales de enfermería, que laboran en el servicio de Emergencia, el $32.50 \%$ tienen un nivel de conocimiento deficiente, solo un $2.5 \%$ tiene conocimiento regular, no se encontraron nivel de bueno y excelente. Asimismo, el nivel de conocimiento de las enfermeras que laboran en el servicio de Cirugía, un $17,50 \%$ tiene conocimiento deficiente sobre el tema en estudio, un $15 \%$ presenta nivel de conocimiento regular, un $12.5 \%$ presenta conocimiento regular y solamente un $2.5 \%$ presenta conocimiento excelente. Del mismo modo, al analizar el nivel de conocimiento de las enfermeras del servicio de Cuidados Intensivos, un 7,5\% tiene conocimiento deficiente, un $10 \%$ evidencia un nivel de conocimiento regular, y no se registraron puntajes en el nivel de conocimiento bueno y excelente.

Comparando los puntajes alcanzados por los profesionales de enfermería, respecto al nivel de conocimientos del PAE sobre el cuidado de la salud espiritual, los enfermeros que laboran en la Unidad de Cuidados Intensivos presenta un nivel de conocimiento más alto, en relación a las otras unidades críticas. 


\section{DISCUSIÓN}

Los resultados obtenidos en las tablas 3 y 4, respecto al nivel de conocimiento de los profesionales de enfermería sobre el Proceso de Atención de Enfermería en el cuidado de la salud espiritual, señalan que el conocimiento de la mayoría de los encuestados es deficiente con puntaje de 57,50\%, en este contexto profesional, se han realizado varias investigaciones sobre el aspecto espiritual de la atención de enfermería. Tal es el caso de (Mc Sherry, (1998), citado por (Collado, 2010), quien realizó un estudio en el cual analizó cómo 548 enfermeras percibían la espiritualidad y proveían cuidado espiritual en la práctica clínica.

Los resultados indicaron que el $71.4 \%$ identificó en el paciente la necesidad espiritual, aunque solo $39.9 \%$ se sentían capacitadas para satisfacer estas necesidades, esto responde a la evaluación realizada en el trabajo de investigación, sobre el nivel de conocimiento del cuidado espiritual, una enfermera poco capacitada en esta área se sentirá a su vez poco capaz de brindar un cuidado integral $\mathrm{y}$, por ende, el paciente no recibirá un cuidado integral. La calidad del cuidado de enfermería demanda hoy en día una interpretación humanística y el respeto por la integridad funcional de los seres humanos, el cuidado del enfermero en el paciente, en fase crítica, se aborda con un enfoque holístico, es decir como un todo integral teniendo en cuenta sus aspectos físicos, intelectuales emocionales, sociales, humanísticos, espirituales y religiosos, dicho enfoque ayudará a mantener su dignidad, su estado de vida, respetar sus creencias, costumbres y filosofía. Del mismo modo, (Espíndula, Ranier, Do, \& Bello, 2010), en un estudio titulado "Religión y espiritualidad: una perspectiva de profesionales de la salud" determinaron que la mayoría de los profesionales de la salud se declaró espiritualista, dos son católicos, un médico se dice budista y una médica Espírita. Creen que la religión es inherente a todo ser humano. Los convictos de sus religiones creen en la protección divina y reconocen la religiosidad como sustento y confort para el paciente y sus familiares, también para que puedan enfrentar la situación de enfermarse. Ellos esperan que esos enfermos vivan su fe con prudencia y siempre adhiriendo a la realidad.

Otro estudio realizado por (Whetsell, 2005) determinó que los niveles más altos de bienestar espiritual tienen niveles igualmente elevados de fortaleza relacionados con la salud. El sexo, la edad, la educación y el estado civil mostraron una relación positiva con los niveles de bienestar espiritual.
Asimismo, en la práctica, las enfermeras tienden a pasar por alto este importante aspecto, tal es así que (Calabria y Macrae, (1994); citado por (Potter, 2004) mencionan: "Es demasiado frecuente en enfermería comprobar que los profesionales no ponen demasiado énfasis en la dimensión espiritual de la naturaleza humana, quizá porque no es demasiado científico o es difícil de medir o cuantificar. Quizás porque hay individuos que no creen en Dios o en un ser superior. Con frecuencia se compara la espiritualidad con la religión y el carácter privado de la orientación religiosa del individuo. Pero la espiritualidad es un concepto mucho más amplio y unificador que la religión". Esto revela la necesidad de un conocimiento adecuado en la enfermera sobre su rol en el cuidado espiritual, especialmente en los momentos de dolor o pérdida de un ser querido.

Se asume por lo descrito anteriormente que el cuidado espiritual no es una opción, sino un elemento constitutivo del cuidado, sin embargo, en la práctica las enfermeras tienden a pasar por alto este importante aspecto, tal es así que (Calabria y Macrae; citado por (Potter, 2004) mencionan: "Es demasiado frecuente en enfermería comprobar que los profesionales no ponen demasiado énfasis en la dimensión espiritual de la naturaleza humana...". Por lo tanto, el cuidado espiritual debe ser ofrecido al paciente en todo momento, con un alto sentido de responsabilidad, dedicación y respeto a todo ser humano que así lo necesite. Además, este cuidado debe ser planificado de acuerdo con las necesidades previamente identificadas durante la fase de estimado y valoración (Collado, 2010). El cuidado espiritual, como las demás áreas, deben ser afrontadas de manera racional y metodológica a través de la aplicación del Proceso de Atención de Enfermería.

Por lo tanto, se hace necesario que así como la enfermera es preparada para los diferentes aspectos de la atención del cuidado de los pacientes, también sea capacitada en el conocimiento del aspecto espiritual para que pueda brindar soporte al cuidado espiritual, del paciente. (Zevallos., 2014) plantea que "la enfermera requiere complementar su habilidad profesional en solucionar problemas, como tener pensamiento crítico, intencionalidad de dar cuidado, y conocer algunas particularidades del cuidado espiritual como el trabajo de fortalecer la esperanza, comprender qué es el cuidado, tener destrezas en la comunicación frente a esta situación de vida y dar manejo integral".

Asimismo (Zevallos., 2014) plantea que "la enfermera requiere complementar su habilidad profesional en solucionar problemas, como tener 
Nivel de conocimiento sobre el Proceso de Atención de Enfermería (PAE) en el cuidado de la salud espiritual de los profesionales de Enfermería que laboran en las unidades críticas del Hospital Nacional Hipólito Unanue, Lima 2010

pensamiento crítico, intencionalidad de dar cuidado, y conocer algunas particularidades del cuidado espiritual como el trabajo de fortalecer la esperanza, comprender qué es el cuidado, tener destrezas en la comunicación frente a esta situación de vida y dar manejo integral". Asimismo, el profesional de enfermería acompaña a la persona en sus experiencias de salud de acuerdo a su evolución, con un sentido de sensibilidad y compromiso moral en el cuidado.

A su vez se puede contrastar con presente trabajo de investigación realizado por García (2007), un estudio titulado "Opinión del paciente con cáncer sobre los cuidados de la enfermera en la satisfacción de sus necesidades emocionales y espirituales en el servicio de medicina oncológica del hospital Belén de Trujillo 2006". Donde la Población está constituida por los pacientes oncológicos que acuden a recibir atención médica y/o quimioterapia en los meses de noviembre y diciembre del 2006 cuyo número ascendió a 50 pacientes, teniendo en cuenta los criterios de inclusión. Las conclusiones a las que llegaron son: La opinión de los pacientes con cáncer sobre dimensión emocional del cuidado de enfermería es satisfactoria recibiendo el paciente atención personalizada con amabilidad, respeto y confianza; sin embargo, en la dimensión espiritual del cuidado de enfermería en su mayoría la respuesta es insatisfactoria. Esto indica que el paciente tiene gran necesidad del cuidado espiritual, según esta percepción del paciente no recibe esa atención que es importante para ellos. En respuesta al trabajo de investigación, el paciente percibe que es incompleta la atención, ya que se obvia una de las áreas consideradas importantes para el paciente.

En un cuidado significativo de apoyo, es importante para la enfermera comprender los conceptos en los que se fundamenta la salud espiritual. Los conceptos de fe, esperanza, bienestar espiritual y religioso proporcionan guías para comprender los puntos de vista que cada individuo tiene de la vida y de su valor.

Según Bermejo (1997), lo espiritual refiere a aquellos aspectos de la vida humana que tienen que ver con experiencias que trasciende los fenómenos sensoriales. No es lo mismo que "religioso", aunque para muchas personas la dimensión espiritual de sus vidas incluye un componente religioso. El aspecto espiritual de la vida humana puede ser visto como un componente integrado junto con los de la vida humana con los componentes físicos, psicológicos y sociales. A menudo se preside como vinculado con la religión. (Kozier, 1999).

Según (Marriner A., 1994), Virginia Henderson, afirma que respetar las necesidades espirituales de los enfermos y asegurar que puedan satisfacerlas en todas las circunstancias, forma parte de los cuidados de enfermería de base.

Si el paciente pierde la esperanza en aquello en lo que cree y le da fortaleza, perderá el ánimo de continuar con su tratamiento, asimismo, se rendirá ante la enfermedad, permitiendo que esa pueda consumirlo y acabe con su vida más rápido, ya que ha perdido aquello que para él es lo que le daba sentido a su vida. Citado por (Kozier, 1999).

Los pacientes pueden tener creencias religiosas y que muchas veces atribuyen a la enfermedad como una alteración espiritual. Es por esto importante que el personal de enfermería aprenda a valorar las creencias del paciente $\mathrm{y}$, siempre que sea posible, a incorporar, dentro del proceso asistencial al paciente, algunos aspectos de curación que formen parte del sistema de creencias del paciente.

El amor, la confianza, la esperanza, el perdón, el significado y la comunidad son necesidades espirituales que todas las personas tienen (Carson, (1989); citado por (Kozier, 1999). Aprender a compartir estas necesidades ayuda a la enfermera a encontrar la forma de prestar cuidado y apoyo espiritual a los clientes.

Se dispone de numerosas acciones de enfermería para ayudar a los pacientes a satisfacer sus necesidades espirituales. Según Bermejo (1997). Un paciente con una buena dosis de salud espiritual encontrará esperanza, significado objetivo y valor en la existencia. (Kozier, 1999).

Bajo la misma concepción, (Salguero, 2004) comenta que la atención espiritual no es un lujo para unos cuantos, es el derecho esencial de todo ser humano, tan esencial como la libertad política, la asistencia médica y la igualdad de oportunidades. En este sentido, un ideal democrático real incluiría, como una de sus verdades esenciales, la atención espiritual experimentada para todos.

Entonces, es necesario que el aspecto del cuidado espiritual en la atención de enfermería no sea obviado por sus implicancias determinantes: ayuda a los pacientes a mantener su fe en un sistema de valores y prácticas e influye en la salud física, psicológica, social y en el desarrollo humano, (Potter, 2004). Por lo tanto, el cuidado espiritual debe ser ofrecido al paciente en todo momento, con un alto sentido de responsabilidad, dedicación y respeto a todo ser humano que así lo necesite. Además, este cuidado debe ser planificado de acuerdo con las necesidades 
previamente identificadas durante la fase de estimado y valoración (Collado, 2010).

\section{Declaración de financiamiento y de conflicto de intereses:}

El estudio fue financiado por la autora, quien declara no tener algún tipo de conflicto de interés en la investigación realizada.

\section{Correspondencia}

María Magdalena Díaz Orihuela

Universidad Peruana Unión

Carretera Central Altura Km. 19.5 Ñaña-Lima. Teléfono: 2010400

Correo electrónico: magi@upeu.edu.pe

\section{REFERENCIAS BIBLIOGRÁFICAS}

Bermejo, J. (1997). Espiritualidad y mayores. Madrid: Centro de Humanización de la Salud. Recuperado de: http://www.gerontologia.org/portal/archivosUpload/ Espiritualidad_Mayores.pdf

Collado, R. (2010). Cuidado espiritual, labor del profesional de enfermería. Revista Universidad Interamericana de Puerto Rico. Recuperado de: http://scholar.google.com/ scholar?hl=en\&btnG=Search\&q=intitle:Cuidado +espiritual,+labor+del+profesional+de+enfermería\#1

Espíndola, J. A., Ranier, E., Do, M., \& Bello, A. A. (2010). Religión y espiritualidad: una perspectiva de profesionales de la salud. Rev. Latino-Am. Enfermagem, 18(6),12291236. Recuperado de: http://www.scielo.br/scielo. php?pid=S0104-11692010000600025\& script $=$ sci abstract\&tlng=es

García (2006), Opinión del paciente con cáncer sobre los cuidados de la enfermera en la satisfacción de sus necesidades emocionales y espirituales en el servicio de medicina oncológica del hospital de Belén. (Tesis de Maestría). Trujillo.

Kozier, E. B. (1999). Fundamentos de enfermería: Concepto, teoría y práctica. México: Editorial MC Graw Hill Interamericana.

Marriner A. (1994). Modelos y teorías de enfermería (p. 356). España: Mosby/Doyma.

Pinedo, M., Rebolledo, D., \& Siles, J. (2009). Cuidado de enfermería en el "sufrimiento espiritual" aplicando el modelo de Jaane Watson. Salud, Arte y Cuidado, 2(2), 8-13. Recuperado de: http://bibvirtual.ucla.edu.ve/db/ psm_ucla/edocs/sac/sac0202/sac020203.pdf

Potter, P. (2004). Fundamentos de enfermería (603-604). España: MC Graw Hill Interamericana.

Salguero, J. M. (2004). Educar Para la Paz: El caso de un país dominado por la violencia. Colombia. (Tesis doctoral). Universidad Complutense de Madrid. Recuperado de: http://biblioteca.ucm.es/tesis/edu/ucm-t27290.pdf

Whetsell, M. (2005). Niveles de bienestar espiritual y de fortaleza relacionados con la salud en adultos mayores. Aquichan, 5(1), 72-85. Recuperado de: http://www. redalyc.org/articulo.oa? $\mathrm{id}=74150108$

Zevallos., Y. M. S. (2014). Significado del cuidado de la dimensión espiritual para enfermeras que asisten personas posoperadas de cirugía cardiovascular. (Tesis de Licenciatura). Universidad Católica Santo Toribio de Mogrovejo. Recuperado de: http://tesis.usat.edu. pe/jspui/bitstream/123456789/181/1/TL_Salazar_ Zevallos_Yngrid.pdf

Recibido: 20/02/2014 Aceptado: 11/10/2014 